\title{
Proliferation of mesenchymal stem cell trials for COVID-19: risks and recommendations
}

\author{
James A Smith ${ }^{1,2,}{ }^{*}$, Roxanna E Abhari ${ }^{1}$, Gary S Collins ${ }^{1,3}$, Andrew J Carr ${ }^{1,2}$
}

1. Botnar Research Centre, Nuffield Department of Orthopaedics, Rheumatology and Musculoskeletal Sciences, University of Oxford, UK

2. National Institute for Health Research Oxford Biomedical Research Centre, John Radcliffe Hospital, Oxford, UK

3. Centre for Statistics in Medicine, Nuffield Department of Orthopaedics, Rheumatology and Musculoskeletal Sciences, University of Oxford, UK

*Author for correspondence: James A. Smith; james.smith2@ndorms.ox.ac.uk; Botnar Research Centre, Nuffield Department of Orthopaedics, Rheumatology and Musculoskeletal Sciences, University of Oxford, OX3 7LD, UK. Twitter: @JamesASmith92

Key words: research quality, meta-research, stem cell based intervention, pandemic, coronavirus, SARS-CoV-2, pneumonia, critical review

\section{Introduction}

At least 20 clinical trials of mesenchymal stem/stromal cells (MSCs) for the treatment of covid-19 have been registered. We assess the emerging trial registrations and argue that the information gain is likely to be low, while the likelihood of false-positives and overinterpretation is high. The rush to test treatments may come at the expense of research quality.

Covid-19 is a global health priority requiring urgent development of therapeutics. As of $6^{\text {th }}$ April 2020, 927 clinical trials for covid-19 had been registered spanning many therapeutic approaches (https://www.who.int/ictrp/en/). One such approach is the use of MSCs. MSCs are thought to have immunomodulatory and anti-inflammatory properties and have been tested in clinical studies for a large range of indications, including acute respiratory distress syndrome. As a result, there is some rationale for their use in covid-19. Understanding whether or not MSCs are useful for covid-19 could clearly be valuable. However, it is important that limited resources are allocated prudently and that patients are not unnecessarily subject to studies that are unlikely to produce reliable information that influences patient care. Here, we characterise and critically assess global trial registrations of MSCs for covid-19, identify areas of concern, and make suggestions for improvements.

\section{Characteristics of clinical trial registrations using MSCs for covid-19}

We identified 20 clinical trials for covid-19 using MSCs registered by $6^{\text {th }}$ April 2020 and extracted data on each of the identified trials (Table 1; supplementary methods and full dataset: https://osf.io/ctsv4/). Across all trials, there were 61 primary outcomes (median: 2; range: $1-20$ ). The trials intend to enrol 984 patients in total and the median (range) total sample size for a trial was $33(5-200)$. Nearly all $(n=19)$ trials were explicitly investigating treatment of covid-19 pneumonia, and most $(n=13)$ used umbilical cord MSCs (UC-MSC). Nearly half the studies $(n=9)$ lacked a control arm, two indicated that some form of blinding 
will be used, and most were single centre $(n=14)$. Most trials with a control arm stated they would randomise patients $(n=9)$, and where the trial phase was stated, it was either phase 0 $(n=3)$, phase I $(n=6), I I(n=4)$ or $I / I I(n=1)$. Unfortunately, much information was poorly reported in the registrations. For example, no information on the timing of any outcome measure was provided in 10 (50\%) of studies, no information on dosing (number of cells or number of doses) was provided in eight (40\%) studies, the route of administration was not provided in four $(20 \%)$ of studies.

Table 1: Characteristics of registered MSC clinical studies for covid-19 (ROA = route of administration)

\begin{tabular}{|c|c|c|c|}
\hline & Characteristic & $\mathbf{N}$ & Percent \\
\hline \multicolumn{4}{|l|}{ Sponsor } \\
\hline & Academic/hospital & 16 & $80 \%$ \\
\hline & Industry & 4 & $20 \%$ \\
\hline \multicolumn{4}{|l|}{ Location } \\
\hline & China & 17 & $85 \%$ \\
\hline & Brazil & 1 & $5 \%$ \\
\hline & Jordan & 1 & $5 \%$ \\
\hline & Not stated & 1 & $5 \%$ \\
\hline \multicolumn{4}{|c|}{ Trial objectives } \\
\hline & Therapeutic vs. preventive ( $\mathrm{N}$ therapeutic) & 20 & $100 \%$ \\
\hline & Investigating covid-19 pneumonia & 19 & $95 \%$ \\
\hline & At least one efficacy primary outcome & 20 & $100 \%$ \\
\hline & At least one safety primary outcome & 4 & $20 \%$ \\
\hline \multicolumn{4}{|l|}{ Intervention } \\
\hline & Dose reported & 12 & $60 \%$ \\
\hline & Intravenous ROA & 16 & $80 \%$ \\
\hline & ROA not stated & 4 & $20 \%$ \\
\hline \multicolumn{4}{|c|}{ Cell source } \\
\hline & Umbilical cord & 13 & $65 \%$ \\
\hline & Not stated & 4 & $20 \%$ \\
\hline
\end{tabular}




\begin{tabular}{|c|c|c|c|}
\hline & Characteristic & $\mathbf{N}$ & Percent \\
\hline & Dental pulp & 2 & $10 \%$ \\
\hline & NestCell ${ }^{\circledR}$ & 1 & $5 \%$ \\
\hline \multicolumn{4}{|l|}{ Trial characteristics } \\
\hline & Blinding stated & 2 & $10 \%$ \\
\hline & Control arm & 11 & $55 \%$ \\
\hline & Randomisation $^{a}$ & 9 & $82 \%$ \\
\hline & Single centre & 14 & $70 \%$ \\
\hline \multicolumn{4}{|l|}{ Phase } \\
\hline & Not stated & 6 & $30 \%$ \\
\hline & Phase 0 & 3 & $15 \%$ \\
\hline & Phase 1 & 6 & $30 \%$ \\
\hline & Phase 1/Phase 2 & 1 & $5 \%$ \\
\hline & Phase 2 & 4 & $20 \%$ \\
\hline Percentage of thos & & & \\
\hline
\end{tabular}

\section{Limited information gain}

There is a mismatch between the aims of these studies, the way they are structured, and information that would be valuable to patients and the scientific community. Small, uncontrolled, or single-centre trials for a new therapeutic approach in a new indication could be useful for investigating safety, but just four studies specified a primary outcome related to safety. Safety of MSCs has already been investigated extensively (Hoogduijn and Lombardo, 2019), including in similar indications (e.g. ARDS), so further safety testing may not be needed. However, since detailed descriptions of the MSC products are generally not specified in the trial registrations, we do not know if the results of previous studies will be applicable here. If the MSC products are different, we must ask what the rationale is for not using those products that have already been shown to be safe.

Unlike safety, efficacy of MSC therapies has proven elusive despite apparently promising early clinical outcomes and preclinical evidence. All 20 registered trials had at least one primary outcome related to efficacy. Though there has been one notable success in the field (Alofisel, by Tigenix, for complex anal fistulas in adults with Crohn's disease, approved by the European Medicines Agency in 2018), there have been hundreds of early phase studies reporting positive results, while results from many phase III clinical studies of MSCs remain unpublished or have been negative (Galipeau and Sensébé, 2018). It is clear that we cannot infer efficacy from early phase trials. Trials designed to reliably test efficacy of MSCs for covid-19 could 
therefore be valuable. Unfortunately, many of the covid-19 trials here do not have a control arm; as this is a new disease, reliable data on its natural progression are not available, and control arms are needed to assess efficacy. Even amongst those that do have a control arm, the majority intend to enrol few patients and it is unlikely that they are appropriately powered to detect plausible differences in efficacy and effectiveness. The largest studies we found could plausibly be large enough to detect differences in efficacy, but closer examination of their characteristics is not reassuring (Table 2).

Table 2: Problems with the largest 4 registered trials by number of patients receiving MSC therapy

\begin{tabular}{|l|l|l|}
\hline $\begin{array}{l}\text { Trial registration } \\
\text { number }\end{array}$ & $\begin{array}{l}\text { Total N } \\
\text { (treatment N) }\end{array}$ & Example of potential problems with the trial \\
\hline ChiCTR2000031430 & $200(100)$ & $\begin{array}{l}\text { Patients will not be randomised to treatment vs control, } \\
\text { leading to potential selection bias, imbalance in patient } \\
\text { characteristics and biased treatment effects. Although } \\
\text { blinding is not stated, the control arm is conventional } \\
\text { treatment so participants and physicians will likely have } \\
\text { knowledge of allocation. There are at least 20 primary } \\
\text { outcomes. }\end{array}$ \\
\hline ChiCTR2000029990 & $120(60)$ & $\begin{array}{l}\text { A paper with results referencing this trial registration } \\
\text { has already been published (Leng et al., 2020), } \\
\text { announcing positive results with MSCs. However, there } \\
\text { is considerable mismatch between the trial registration } \\
\text { and the publication: for example, there are 7 patients in } \\
\text { the treatment arm in the paper, but 60 in the treatment } \\
\text { arm in the registration. }\end{array}$ \\
\hline NCT04288102 & $90(60)$ & $\begin{array}{l}\text { Trial participants are allocated in a 2:1, treatment: } \\
\text { control allocation ratio, resulting in a larger required } \\
\text { sample size to achieve a particular level of power } \\
\text { (MacPherson and Kimmelman, 2019). }\end{array}$ \\
\hline NCT04315987 & $66(66)$ & $\begin{array}{l}\text { This study has no control group, despite aiming to assess } \\
\text { efficacy. }\end{array}$ \\
\hline
\end{tabular}

Meta-analysis is also unlikely to be useful in this context. First, it is generally only possible for randomised controlled trials (RCTs), and the number of patients in suitable trials $(n=9)$ would be limited to approximately 564 ( $57 \%$ of total). Second, there is substantial variation in the outcome measures used across studies, which would further limit the ability to combine results: for example, there is no common primary outcome across the two largest RCTs ( $n=120$ and $n=90$ ), and the number of primary outcomes being investigated across the trials is also concerning. Third, there is variation in the cells used which may limit comparability: most studies use UC-MSCs, but one study reported use of dental pulp MSCs, and within those studies using UC-MSCs there is likely further variation in the specific product or preparation. For four trials, we do not yet have any information about cell source, and for eight trials, no information on dosage is provided. Finally, meta-analysis relies on the availability of accurate descriptions of the methods and findings of clinical studies, and bias in the reporting or conduct of trials can limit its use. As discussed below, emerging literature indicates potential problems. Many of these issues have been noted when MSC therapy meta-analyses have been attempted previously (e.g. Dave et al., 2015). 
In our view, it is likely that further trials will be needed for reliable conclusions about efficacy of MSCs for covid-19. Unfortunately, there is great redundancy across trials but little standardisation: many are using similar cell types for the same indication, and it is unclear why are so many trials needed to answer similar questions. Additionally, registrations often do not include information essential for evaluating the trial, which may reduce confidence in reported results. The value of these studies must be questioned.

False positives and the emerging literature

In the current rush to find a treatment for covid-19, there is a risk of over- or misinterpretation of positive efficacy results from early studies. Even in the absence of any bias (e.g. selective reporting), if we assume that all of the covid-19 trials analysed here would claim a positive effect of treatment at $\alpha=0.05$ and that there is in fact no true effect, the probability of at least one significant finding across the 20 trials is 0.64 (i.e. 1- $0.95^{\wedge} 20$ ). Given that many of the studies are uncontrolled, not blinded, have different study designs, and have multiple primary outcomes, there is ample opportunity for bias, which along with small sample sizes, can dramatically increase the chance of misleading conclusions. Furthermore, even if there is some true effect of MSCs in this context, the reported effect size will likely be inflated due to the small sample sizes and therefore probable low power of the studies (Ioannidis, 2008).

Publications about MSCs for covid-19 do not provide much reassurance regarding interpretation of early findings or the quality of literature that may result from these trial registrations. At least two clinical investigations of MSCs for covid-19 pneumonia have been published. However, they have significant limitations: one included only a single patient (Liang et al., 2020) and the other had a total of ten patients with seven in the treatment arm (Leng et al., 2020). Worryingly, the latter publication diverged considerably from the sample sizes (amongst other things) stated in the clinical trial registration ( $n=60$ per arm; registration number ChiCTR2000029990). It is worth reflecting on whether the results in these first 7 treated patients would have been published if the findings were negative. It seems that even those trial registrations that describe (relatively) good quality trials may produce unusable or misleading data. Despite the limitations, commentaries have noted the "remarkable new data using MSC" (Metcalfe, 2020), and their "promise for combating coronavirus"(Shetty, 2020). A Mesoblast press release announcing evaluation of Remestemcel-L for covid-19, citing Leng et al., stated: "MSCs cured or significantly improved functional outcomes in all seven treated patients" (http://investorsmedia.mesoblast.com/static-files/c1428818-0b9f-44f9-bb4f79ad518002cc).

Such statements, taken in the context of the quality of the available evidence, may generate false hope for as yet unproven therapies, and the risk of misleading or unreliable findings appears particularly great in the context of MSC therapy. Permissive regulatory pathways for regenerative medicines, such as those in Japan, India and Taiwan, allow therapies to be marketed with evidence of safety but limited evidence of efficacy. Indeed, MSC therapies have previously entered the market with limited demonstrated efficacy (e.g. Prochymal for children with acute graft versus host disease by Osiris Therapeutics, in Canada). MSCs are a poorly defined and contested cell type, yet they are very accessible: many hospitals and companies have the ability to manufacture or expand MSCs of some description. Without accurate and detailed reporting of specific cell products tested, positive results in a trial may 
be interpreted as indicating that MSCs in general have an effect. Hospitals might then make or source MSCs for compassionate use without regulatory approval being necessary (some are already arguing for this (Atluri et al., 2020)). Alternatively, patients may directly access stem cell "therapies" from unregulated or lightly regulated clinics which continue to be problematic even outside of the panic caused by a pandemic. Premature clinical application of stem cell based therapies and patients seeking unproven stem cell therapies outside of trials have previously been highlighted (e.g. MacPherson and Kimmelman, 2019).

\section{Recommendations}

The quality of trial registrations could be improved considerably. Several key items (e.g. dosing) are often not stated, and improving the quality of the information in the registrations would make the registrations more useful. When completed, the findings of trials identified here should be accurately and completely reported to allow fair assessment of the quality of the trials, facilitate evidence synthesis, and limit publication bias. As new trials continue to be registered and conducted, better coordination and standardisation would increase the possibility of evidence synthesis (for example, the use of the same primary outcome measures). Duplication of effort may be reduced by searching trial registries for similar studies. Living systematic reviews (e.g. https://www.iddo.org/research-themes/covid19/live-systematic-clinical-trial-review) will also be useful for these purposes once available.

Ideally, appropriately powered, multi-centre studies would be planned that can answer efficacy questions reliably. If resources are not available to conduct such trials, it may be advisable not to proceed with new studies, and the value of additional, small scale, uncontrolled or non-randomized studies should be questioned by investigators and ethics committees. Promisingly, Mesoblast has announced a 240 patient, multi-centre, randomised, placebo-controlled trial to test MSCs for ARDS in covid-19 (http://investorsmedia.mesoblast.com/static-files/e63bf0d5-7dd5-46c0-8381-

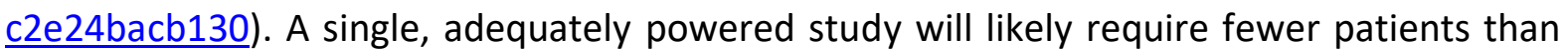
those needed for the large number of smaller trials we have found, and will produce much more robust findings. Such trials in China, where the majority of trials we identified are being conducted, would be valuable.

Our findings add to recent literature highlighting problems with emerging covid-19 research. For example, a living systematic review of covid-19 prediction models (Wynants et al., 2020) found that models are rapidly entering the literature, but that all are at a high risk of bias and probably report optimistic performance, limiting their use. Problems with research into diagnosis using computed tomography have also been noted, prompting calls to "[not] rush the science" (Hope et al., 2020). The same message applies here. Though the desire to rapidly identify and test treatments is admirable, it must be balanced against the quality of the evidence that will be produced. Many trial registrations we have identified are, in our view, likely to produce largely unusable results.

If positive results do emerge from these studies, they must be interpreted in the context of the details of the trial registration, the portfolio of parallel trials in this space, and the historical context of successful early phase studies leading to limited efficacy in later phase trials and few product approvals. Authors of academic articles and the media should be careful to accurately communicate findings to the public and not overstate the promise of 
findings without critical evaluation. Until sufficiently powered, randomised controlled trials or meta-analyses demonstrate a benefit of MSC for covid-19, positive findings should be interpreted with great caution.

\section{Acknowledgements}

JAS receives funding from the National Institute for Health Research (NIHR) Oxford Biomedical Research Centre (BRC) and this study was conducted as part of his post-doctoral research. GSC was supported by the NIHR Biomedical Research Centre, Oxford, and Cancer Research UK (programme grant: C49297/A27294). The views expressed are those of the author(s) and not necessarily those of the NHS, the NIHR, or the Department of Health.

Data and code availability

Data and code are freely available here: https://osf.io/ctsv4/

\section{Declaration of interests}

The authors declare no competing interests.

\section{Bibliography}

Atluri, S., Manchikanti, L., and Hirsch, J.A. (2020). Expanded Umbilical Cord Mesenchymal Stem Cells (UC-MSCs) as a Therapeutic Strategy in Managing Critically III COVID-19 Patients: The Case for Compassionate Use. Pain Physician 23, E71-E83.

Dave, M., Mehta, K., Luther, J., Baruah, A., Dietz, A.B., and Faubion, W.A. (2015). Mesenchymal Stem Cell Therapy for Inflammatory Bowel Disease: A Systematic Review and Meta-analysis. Inflamm Bowel Dis 21, 2696-2707.

Galipeau, J., and Sensébé, L. (2018). Mesenchymal Stromal Cells: Clinical Challenges and Therapeutic Opportunities. Cell Stem Cell 22, 824-833.

Hoogduijn, M.J., and Lombardo, E. (2019). Mesenchymal Stromal Cells Anno 2019: Dawn of the Therapeutic Era? Concise Review. STEM CELLS Translational Medicine 8, 1126-1134.

Hope, M.D., Raptis, C.A., and Henry, T.S. (2020). Chest Computed Tomography for Detection of Coronavirus Disease 2019 (COVID-19): Don't Rush the Science. Ann Intern Med.

Ioannidis, J.P.A. (2008). Why Most Discovered True Associations Are Inflated. Epidemiology $19,640-648$.

Leng, Z., Zhu, R., Hou, W., Feng, Y., Yang, Y., Han, Q., Shan, G., Meng, F., Du, D., and Wang, S. (2020). Transplantation of ACE2-mesenchymal stem cells improves the outcome of patients with COVID-19 pneumonia. Aging and Disease 11, 216-228.

Liang, B., Chen, J., Li, T., Wu, H., Yang, W., Li, Y., Li, J., Yu, C., Nie, F., and Ma, Z. (2020). Clinical remission of a critically ill COVID-19 patient treated by human umbilical cord mesenchymal stem cells. ChinaXiv.

MacPherson, A., and Kimmelman, J. (2019). Ethical development of stem-cell-based interventions. Nature Medicine 25, 1037-1044. 
Metcalfe, S.M. (2020). Mesenchymal stem cells and management of COVID-19 pneumonia. Medicine in Drug Discovery 5, 100019.

Shetty, A.K. (2020). Mesenchymal Stem Cell Infusion Shows Promise for Combating Coronavirus (COVID-19)- Induced Pneumonia. Aging Dis 11, 462-464.

Wynants, L., Calster, B.V., Bonten, M.M.J., Collins, G.S., Debray, T.P.A., Vos, M.D., Haller, M.C., Heinze, G., Moons, K.G.M., Riley, R.D., et al. (2020). Prediction models for diagnosis and prognosis of covid-19 infection: systematic review and critical appraisal. BMJ 369. 